\title{
Memoria y modos de representar los juicios contra genocidas en Argentina. Un concepto para abordar la prensa escrita
}

\author{
Memory and ways to represent judgments against genocides in Argentina. \\ A concept to analyze the written press
}

(iD) Natalia Paola Crocco ${ }^{1}$

\begin{abstract}
Resumen
En el presente artículo se propone realizar un recorrido conceptual de distintos trabajos académicos que han tematizado y conceptualizado la temática de la "memoria". Ello con el objetivo de construir un concepto para abordar el modo en que la prensa escrita representa al proceso de juzgamiento contra los responsables de los crímenes cometidos por el Estado durante la última dictadura cívico militar en Argentina (1976-1983). El trabajo comienza con una introducción que sitúa a la investigación en el marco del proceso de juzgamiento en Argentina como el acontecimiento que permite la emergencia de nuevos discursos y sentidos vinculados al terror, produciendo novedades en los desarrollos de las ciencias sociales en general y los estudios de memoria en particular. El primer apartado se aboca a los modos en que el concepto de memoria ha sido tematizada y definida en tanto categoría analítica por las Ciencias Sociales. El segundo, tercer, cuarto y quinto apartado tienen por objetivo acercar las principales características del concepto de memoria construido a lo largo del artículo, en ellos se da cuenta de la memoria como un proceso encuadrado, conflictivo, múltiple, de larga duración y que conecta pasado, presente y futuro. Por último, la discusión y las conclusiones pretenden integrar el concepto construido a lo largo del texto con el abordaje de los juicios en la prensa escrita y dejar abiertos algunos interrogantes.
\end{abstract}

Palabras clave: Memoria; juicios; prensa escrita

\begin{abstract}
This article proposes to review and discuss several academic works on the concept of "memory". The main aim is constructing a frame to deal with the way in which the graphic press represents the trial process against those responsible for the crimes committed by the State during the last civic-military dictatorship in Argentina (19761983). The article opens with an introduction that places the trial process in Argentina as an event allowing for the emergence of new discourses and meanings linked to terror, which in turn produces new developments in the Social Sciences in general and in the studies of memory in particular. The first section focuses on the ways in which "memory" has been discussed and defined as an analytical category by the Social Sciences. The subsequent sections
\end{abstract}

Tipología: Artículo de reflexión

Recibido: $13 / 04 / 2017$

Evaluado: 22/05/2017

Aceptado: 26/05/2017

Disponible en línea: 01/07/2017

Como citar este artículo: Crocco, N.P. (2017). Memoria y modos de representar los juicios contra genocidas en Argentina. Un concepto para abordar la prensa escrita. Jangwa Pana, 16 (2), 282 - 291. DOI: http://dx.doi.org/10.21676/16574923.2134

1. M.Sc. en Investigación en Ciencias Sociales. Universidad de Buenos Aires. Argentina. Correo electrónico: crocconatalia@gmail.com. ORCID ID: 0000-0001-8647-5506 
aim to coalesce the main characteristics of the concept of memory built throughout the article, thus understood as a situated, conflictive, multiple, long-lasting process that connects past, present and future. Finally, the discussion and conclusions seek to contrast such concept with the approach of trials in the written press, leaving some questions open to further debate.

Keywords: Memory; Trials; written press

\section{Introducción}

$\mathrm{D}$ esde el año 2005, se desarrolla en Argentina un proceso de juzgamiento penal a gran escala hacia los responsables y ejecutores del genocidio perpetrado en el país entre los años 1975 y 1983. Esta instancia fue posible luego de la anulación de las leyes de Punto Final (1986) y Obediencia Debida $(1987)^{2}$ en el año 2003 y su posterior declaración de inconstitucionalidad por parte de la Corte Suprema de la Nación en el año 2005. Se ubica la cronología del genocidio en febrero de 1975 con la puesta en marcha del decreto que ordena el Operativo Independencia en la provincia de Tucumán. El mismo se lleva a cabo durante el gobierno constitucional de Isabel Martínez de Perón y no en marzo de 1976 con el inicio de la dictadura. Este señalamiento se funda en que a partir de la ocupación de la provincia de Tucumán por parte del Ejército comienza a desplegarse en forma planificada y sistemática la práctica represiva que luego a partir del golpe de estado se haría extensiva a todo el territorio nacional; secuestros, torturas, reclusión en centros clandestinos de detención y la desaparición de personas como tecnología de poder represiva predominante.

Los juicios han tomado gran relevancia tanto a nivel nacional como internacional; esto se debe a que configuran un caso excepcional en el cual se juzga a los responsables de los crímenes cometidos por el estado en el contexto jurídico nacional, con jueces naturales -no especiales-,

2. Estas dos leyes pusieron un coto a las responsabilidades de las fuerzas armadas para aquellos miembros que cumplían órdenes dentro de la cadena de mando (Obediencia Debida) e introdujo un límite temporal de treinta días para poder realizar denuncias instaurando la extinción de la acción penal luego de ese período de tiempo. en el fuero penal federal, sin la intervención de tribunales ni organismos internacionales. Es decir, es el propio estado el que juzga a los crímenes que otrora cometió.

La apertura de los juicios desbloquea el largo período de impunidad imperante desde fines de la década de 1980, posibilitando la emergencia de nuevos discursos, narrativas y representaciones sobre un pasado vinculado con el terror. Se generan novedosos desafíos, especialmente para los estudios abocados a la memoria social y las ciencias sociales que se relacionan con los modos de pensar este pasado que reaparece en el presente y se reactualiza a partir de los procesos judiciales. Un desafío que, planteado en los términos de Henry Rousso, se vincula a los efectos a largo plazo de un acontecimiento traumático del pasado reciente y que hace discutir fuertemente con la perspectiva que supone a los acontecimientos como delimitados en el tiempo; "un pasado que no pasa" (2000) y que actualiza en el presente sus quiebres y traumas. Otros autores han reflexionado en torno a cómo denominar estos procesos vinculados al terror. En este sentido, otras aproximaciones posibles para denominar a estos procesos son; catástrofe social (Puget, 2006); experiencia límite (Pollak, 2006) y catástrofe del sentido (Gatti, 2008). En esta línea, el interrogante que ronda a este proceso institucional que acumula diez años y se vincula estrechamente a la memoria social es; ¿cómo gestiona una sociedad su pasado?, ¿qué actores y soportes institucionales intervienen en esta gestión?

No es la primera vez que las ciencias sociales y el campo específico de los estudios sobre memoria se encuentran frente a este dilema. Desde la década de 1980, tanto en Argentina como en gran parte de 
América del Sur ha sido muy profusa la producción académica al respecto; “(...) los debates acerca de la memoria de períodos represivos y de violencia política son planteados con frecuencia en relación con la necesidad de construir órdenes democráticos en los que los derechos humanos estén garantizados para toda la población (...)" (Jelin, 2002, p. 11).

Una vez afianzadas estas democracias, los debates en torno a la memoria se reconfigurarán dependiendo de la coyuntura política y social. En este sentido, los juicios plantean nuevos debates y desafíos para reflexionar sobre los procesos de memoria social, que involucran a actores sociales que forman parte de la construcción y la transmisión de representaciones y sentidos sobre este nuevo acontecimiento de la historia Argentina.

Un actor fundamental que interviene en los modos en que se configura la memoria social sobre los juicios es la prensa escrita ${ }^{3}$. Algunos medios de comunicación realizan una cobertura periodística de las audiencias judiciales, las que utilizan como principal insumo para la publicación de artículos, crónicas y editoriales relacionados a la temática, colocando al juzgamiento a represores en un escenario de visibilidad. Durante el Juicio a las Juntas (1985) se destacó como principal antecedente "El diario del Juicio", una publicación semanal de la editorial Perfil, el cual se abocó exclusivamente a reponer la cotidianeidad de los estrados a partir de múltiples estrategias en sus 36 ejemplares editados entre mayo de 1985 y enero de 1986. Algunas de ellas fueron la publicación de; testimonios de sobrevivientes a la experiencia concentracionaria, entrevistas a los magistrados intervinientes, artículos de autoridad por parte de expertos en materia jurídica y de los organismos de derechos humanos, presentación de información que involucraba directamente a los imputados y la divulgación del alegato fiscal y la sentencia del juicio en forma completa. Este diario fue una

\footnotetext{
3. Este trabajo forma parte de una investigación más exhaustiva en el marco de mi tesis de maestría que tiene como objetivo fundamental analizar y comprender las representaciones que construyen los diarios Clarín, La Nación y Página 12 sobre los juicios a represores en la provincia de Buenos Aires y la Ciudad Autónoma de Buenos Aires en el período comprendido entre los años 2005 y 2015.
}

excelente reposición y transmisión de lo ocurrido en el tribunal que juzgó a las juntas militares ya que si bien las audiencias eran públicas, la televisión cumplió un rol menor debido a que solo se transmitía diariamente tres minutos de imagen sin audio por lo cual fue uno de los principales soportes de transmisión.

Veinte años después, los modos en que se representa a los juicios y a los crímenes a los que estos refieren involucran otras problemáticas no presentes en la década de 1980. Surgen interrogantes y formas de narrar la experiencia muy distintas que se ubican en una nueva disposición de lo que Crenzel (2007) denomina "régimen de memoria", un conjunto de nuevas proposiciones que "organizan el debate público, se convierten en objeto privilegiado de las luchas por dotar de sentido el pasado, y moldean, incluso delimitan, las interpretaciones divergentes" (p. 25). En este sentido, en el presente artículo se propone construir una noción de memoria que permita abordar los sentidos y representaciones construidas por la prensa escrita que pueda dar cuenta de las tensiones y las reconfiguraciones representacionales en el contexto de juzgamiento $\mathrm{y}$ que forman parte de las disputas por otorgar sentidos al pasado.

El objetivo de los siguientes cinco apartados será justamente la construcción de este concepto propio de memoria a partir de un análisis detallado que recupera los principales aportes académicos sobre la temática. En el primero de ellos se realiza un recorrido sobre los modos en que fue definida por las Ciencias Sociales la memoria en tanto categoría analítica. En el segundo apartado se presentan los abordajes de "encuadramiento" de la memoria. En la tercera sección se define a los procesos de memoria como conflictivos, múltiples y en movimiento. En la cuarta, se la caracteriza como un proceso de larga duración. El quinto apartado, íntimamente vinculado al cuarto, pretende abordar los procesos de memoria anudando pasado presente y futuro. Por último, tanto la discusión como las conclusiones tienen como finalidad poner en discusión la reconstrucción de la noción de memoria realizada a lo largo del trabajo con el tratamiento periodístico de los juicios realizado 
por los diarios, retomar los objetivos principales del artículo y dejar un conjunto de interrogantes abiertos para futuras indagaciones.

\section{Los estudios sobre memoria}

La memoria en tanto categoría analítica de las ciencias sociales fue tematizada y definida de diversos modos y perspectivas. El campo de estudios comienza a delimitarse en Europa en la década de 1970 a partir de un conjunto de trabajos provenientes principalmente del campo de la historia. Estos trabajos debaten e interrogan sobre los efectos a largo plazo de acontecimientos traumáticos, principalmente la Segunda Guerra Mundial, la experiencia de ocupación de algunos países europeos durante el transcurso de ésta y el genocidio perpetrado por el nazismo (Portelli, 2003; Lacapra, 2007; Rousso, 2012; Traverso, 2012).

En América Latina, al igual que en Europa, los estudios sobre memoria se plantearon principalmente avanzar en la comprensión de procesos políticos y sociales vinculados a la represión estatal y a la difusión del terror que sucedieron en la región a partir de mediados de la década de 1960; "estudiando las luchas por interpretar y redefinir sentidos acerca de esos períodos del pasado, y situando actores e instituciones involucradas en la tarea de recordar y hacer recordar a otros/as las experiencias de sufrimiento" (Feld, 2012, p. 151). Es decir, es un campo de estudios que no solo se posiciona desde un objetivo de comprensión erudita o académica sino que se tiene un fuerte compromiso social, de "militancia del recuerdo" a partir de la comprensión de lo sucedido.

Entonces, la producción de trabajos sobre memoria social se relaciona íntimamente a la comprensión de las formas de funcionamiento de los procesos de representación de la realidad. Es posible señalar los estudios de Maurice Halbwachs como su principal influencia. Estos trabajos han sido abordados en forma pionera desde los marcos o cuadros (cadres) sociales que posibilitan la construcción de una memoria colectiva, marcos que son portadores de las representaciones del mundo en función de las necesidades y valores de una sociedad (2004). Hay trabajos que siguiendo a Halbwachs se proponen establecer la matriz grupal dentro de la cual se ubican los recuerdos individuales (Namer 1998; Jelin, 2002). Otros autores se han propuesto comprender los modos en que la memoria se instituye a partir de narrativas (Ricoeur 2008; Lacapra, 2007; White, 2003; Portelli 2003; ValensI 2003), han intentado delinear cuestiones en torno a lo que denominan "abuso" de la memoria (Todorov, 2000), "sobreabundancia" (Nora, 1998) y "obsesión” (Traverso, 2012), entre otros.

En América Latina los estudios sobre memoria han sido abordados principalmente por Elizabeth Jelin que entiende a las memorias como procesos subjetivos enmarcados socialmente; para la autora las memorias son espacios de disputa que se encuadran históricamente (2002). Si bien la discusión de esta temática transito múltiples aristas, son pocos los trabajos que se propusieron analizar las representaciones elaboradas por los medios de comunicación en relación al genocidio en Argentina ${ }^{4}$ desde una perspectiva que involucre una visión de los modos en que se representa el pasado desde el presente a partir de una perspectiva de de los modos en que se constituye socialmente la memoria.

Este trabajo se sitúa en este territorio de indagación y se propone la construcción de un concepto de memoria que integre desde una perspectiva sociológica los modos en que la prensa escrita representa a los juicios contra represores.

\section{Primera aproximación. Un concepto de memoria "encuadrado"}

Maurice Halbwachs fue el pionero en tematizar a la memoria en tanto objeto de investigación. Desde la sociología planteó la existencia de cuadros (cadres) sociales de la memoria que son portadores de representaciones del mundo, de

\footnotetext{
4. Quien avanzo a partir de un análisis de la televisión ha sido Claudia Feld, al respecto puede consultarse "Del estrado a la pantalla. Las imágenes del juicio a los ex comandantes en Argentina", Buenos Aires y Madrid, Siglo XXI Editores, 2002.
} 
una cosmovisión coherente a los valores de una sociedad (2005). A partir de estos desarrollos, el objetivo de Halbwachs fue dar cuenta que las memorias individuales siempre se encuentran encuadradas socialmente por estas referencias sociales. Si bien los estudios sobre memoria no surgen con los desarrollos de este autor, estos serán de gran aporte para la configuración posterior del campo, especialmente para aquellos trabajos que intenten delinear la matriz social en la cual se sitúan las memorias o recuerdos individuales (Namer, 1998; Lavabre, 1998; Jelin, 2002). Dentro de este grupo, Marie-Claire Lavabre (1998), en sus comentarios a la noción de memoria colectiva de Halbwachs expondrá que:

El grupo de pertenencia del individuo (el individuo aislado es una ficción) le entrega los instrumentos para reconstruir su pasado y le proporciona los calendarios y las palabras que expresan el recuerdo, así como las convenciones, los espacios y las duraciones que dan su significado al pasado. (p.8)

Retomando estos aportes, se delimitará la primera característica de la noción de memoria que adoptaremos. A la hora de reflexionar sobre los modos en que los medios construyen determinadas representaciones y sentidos sobre los juicios, inevitablemente se debe recurrir al contexto, la coyuntura política y social en la que estas representaciones son posibles y que proveen a los distintos actores (dentro de ellos los medios) de las pautas de posibilidad de enunciación o los "marcos" en los que situar estas representaciones. En este sentido, podemos señalar que en lo que respecta a los modos de pensar y representar a los crímenes cometidos por el estado, las pautas que hacen posible determinadas formas de decir han sido diversas dependiendo de las coyunturas.

Se puede delimitar un período que se inicia una vez finalizada la dictadura en el que los marcos sociales de referencia estuvieron fuertemente vinculados a una lógica discursiva alrededor del "Nunca Más" (CONADEP, 2003). Crenzel (2007) desarrolla que en este marco se desplegaron las iniciativas del gobierno radical para juzgar los crímenes y se articuló una narrativa humanitaria para su denuncia. Dentro de este encuadramiento o "régimen de memoria" (Crenzel, 2007) resulta importante marcar un límite que luego a partir de 2005 marcará un desplazamiento -y se observará en los discursos de los diarios- en términos de lo representable. Este límite puede señalarse en la dicotomía democracia-dictadura que invisibilizó tanto la responsabilidad política del gobierno peronista (1973-1976) como así también de la sociedad civil y las Fuerzas Armadas sobre la existencia de desapariciones anteriores al golpe de estado (Crenzel, 2007). Por último, la construcción de la "inocencia" de la sociedad civil y los desaparecidos (Crenzel, 2007; Feierstein, 2007) es un elemento importante que desde 2005 también será reconfigurado a partir de la emergencia de la recuperación de la militancia como parte de la identidad y la práctica política de quienes testimonian en los juicios como así también la emergencia de la figura de la "complicidad civil".

Entre los años 1989 y 1990, a comienzos del mandato del presidente Carlos Saúl Menem se sancionan un conjunto de diez decretos en los que se indultó a civiles y militares que cometieron crímenes durante la dictadura, incluyendo a los miembros de las juntas condenados en el Juicio a las Juntas y los líderes de las organizaciones políticas armadas. Estos decretos inauguran un período en el que primará una situación de impunidad para los perpetradores de miles de secuestros, torturas, violaciones y desapariciones. En este contexto los marcos de lo memorable se estrechan y la represión y sus consecuencias siguen siendo movilizadas de forma minoritaria por los organismos de derechos humanos y los afectados directos de la represión. En lo que respecta a los medios de comunicación, casi no se trataba el tema; exceptuando el diario Página 12 que desde su aparición a fines de la década de 1980, dio un espacio muy importante al tratamiento de los reclamos de memoria, verdad y justicia, principalmente publicando artículos sobre los juicios de apropiación de menores que se llevaron adelante en la década de 1990 -por ser junto a los delitos contra la propiedad los que quedaron fuera de la legislación de impunidad- y 
luego a fines de la década de 1990 otorgándole visibilidad a los Juicios por la Verdad ${ }^{5}$.

\section{Segunda aproximación. Conflicto, movimiento y multiplicidad de "memorias"}

Retomando el rodeo anterior, específicamente en lo que respecta a estas memorias no correspondidas con los marcos hegemónicos de lo memorable, Michael Pollak (2006) incorpora una perspectiva de análisis que puede delinearse a partir del juego entre la "memoria oficial" y lo que denomina como "memorias subterráneas". Esta dicotomía es productiva para pensar a las memorias prohibidas o silenciadas pero a la vez problemática y polémica $^{6}$. A partir de este juego Pollak (2006) incorpora el elemento del conflicto -no presente en Halbwachs-, el cual aquí se retoma para la construcción del concepto propio de memoria al igual que el elemento de la multiplicidad; no hay memoria colectiva única sino "memorias". Es en este territorio de conflicto, movimiento y multiplicidad que se construyen y configuran las disputas entre la memoria oficial y las memorias subterráneas a la espera de reconfiguraciones coyunturales que posibiliten su emergencia.

\footnotetext{
5. Los Juicios por la Verdad fueron una experiencia que surgieron a fines de la década de 1990 en la ciudad de La Plata en un contexto de impunidad. Los mismos no tuvieron consecuencias penales sino que pretendieron avanzar en la investigación sobre las desapariciones. Gran parte de la investigación realizada en el marco de estos juicios luego fue retomada y utilizada como prueba en los juicios a partir del año 2005. 6. Vale la pena interrogarse que sucedería por ejemplo en casos como el argentino que durante más de diez años (2003-2015) la memoria oficial retomó las consignas históricas de muchos organismos de derechos humanos y además hizo de los derechos humanos una de sus principales políticas de estado. En un contexto donde la memoria oficial intenta elaborar el pasado traumático a partir del reconocimiento de los crímenes de estado y con políticas activas tanto de reparación como de señalamiento de espacios que funcionaron como centros de detención para la represión, donde se declaró un feriado nacional en conmemoración al golpe de estado y se incorporo a los contenidos de la currícula educativa la reflexión específica de los hechos. Qué sucedería en este contexto, vale la misma carátula de memoria subterránea para aquellas que por ejemplo quisieron visibilizar organizaciones defensoras de militares o familiares de represores. En este sentido el concepto de memoria subterránea plantea el interrogante para reflexionar si todas las memorias deben considerarse igual solo por su posición en la correlación de fuerzas entre el par "oficialidad/subterraneidad".
}

Haciendo hincapié en este aspecto de la multiplicidad de la memoria, resulta útil para nuestro concepto incorporar los análisis de Alessandro Portelli (2003). Este autor, destaca que un mismo acontecimiento histórico puede ser interpretado de distintos modos dependiendo quienes sean los actores que realizan la lectura, portadores de identidades divergentes, contrastando los hechos históricos con los relatos de la memoria oficial y realizando un interjuego a partir de la incorporación de la memoria de testigos del acontecimiento quienes a partir de sus subjetividades pueden refutar a la historia oficial.

Desde estos dos autores es posible señalar que la memoria colectiva es fragmentaria e incoherente, $\mathrm{y}$ es en los intersticios de esta fragmentariedad y no coherencia se articula el par recuerdo/olvido en torno a lo que Pollak denomina "experiencias límite". La construcción del recuerdo y el olvido tiene anclaje en el presente, un presente vinculado al pasado por el componente traumático implicado en este tipo de experiencias, y que a su vez, es de suma provisoriedad ya que se aferra en una actualidad que se modifica constantemente, dada la multiplicidad de actores que intervienen en el proceso de constitución de las memorias. Entonces, de esta perspectiva rescataremos fundamentalmente la consideración de las memorias a partir de los conflictos y luchas con el objetivo de poder dar cuenta de la participación activa de los distintos actores (en este caso los periódicos) en tanto productores de distintos sentidos.

\section{Tercera aproximación. Procesos de larga duración}

Recogiendo como disparador estas experiencias límite, es posible interrogarse; ¿por qué algunos acontecimientos del pasado perviven y siguen teniendo efectos en el presente?; ¿de qué particularidades son portadores determinados eventos que los convierten en procesos de larga duración, aún décadas después de haber finalizado?

Pierre Nora (2004) intenta avanzar sobre el primero de estos interrogantes a partir de las indagaciones 
en torno a lo que denomina como lugares (lieux) de memoria de la identidad nacional francesa. Los mismos son entendidos dentro de una acepción amplia en la que se halla "anclada la memoria colectiva y en una vasta topología de la simbología francesa" cuya razón de ser es bloquear el olvido a partir de mantener un estado de cosas. Ejemplo de estos señalamientos son las fechas patrias, los monumentos conmemorativos, el himno nacional, entre otros. A los fines de este trabajo, este avance de Nora constituye un antecedente que permite pensar la permanencia de un estado de cosas a lo largo del tiempo pero que excede ampliamente a nuestro objeto de memoria que se vincula a un hecho histórico traumático. Excede los límites de la conceptualización que se quiere plantear ya que Nora se encuentra interesado en las formas en las que el pasado permanece en el presente, a partir de su fijación o anclaje, y no en lo que se señaló fundamental para este trabajo, que es el cambio y movimiento de las representaciones que constituyen a las memorias.

En esta línea, Henry Rousso se interesa por los desplazamientos, las rupturas y los traumas que determinados acontecimientos generan en una sociedad. Este autor, toma un caso para observar como repercutió en la conciencia nacional francesa; la ocupación alemana de Francia bajo el régimen de Vichy durante la Segunda Guerra Mundial. Toma la "historia" de este recuerdo a partir de la ampliación de los horizontes cronológicos de este acontecimiento. Desde esta perspectiva que plantea la permanencia de un recuerdo y sus desplazamientos a lo largo del tiempo realiza un señalamiento muy interesante a incorporar a nuestro concepto de memoria; la historia de la memoria colectiva es un objeto en sí mismo que quiebra epistemológicamente respecto a otros modos de hacer historia. Con esto quiere plantear un cambio radical respecto a otras formas de investigación histórica ya que plantea que el historiador (es posible trasladar esta figura a una más amplia, la del investigador) para comprender cabalmente la historia -y sus eventos específicos-, debe comprender las diversas representaciones sociales (cuadros sociales retomando la primera aproximación) presentes en la sociedad sobre ese evento y no debe como otrora, limitarse a indagar los sentidos y las representaciones presentes en la historiografía (Rousso, 2012).

En contraposición a la noción de "lugares de memoria", Rousso (2000) definirá el concepto de "vector de memoria", precisamente porque la palabra "vector" da noción de cambio de estado. Los libros de historia, el cine, los actos conmemorativos, la literatura, las producciones periodísticas sirven de ejemplo que dan de manifestaciones que se modifican y los modos en que lo hacen. Respecto a esta perspectiva hay un último señalamiento interesante a resaltar. Rousso (2000) -y también Pollak (2006)- al hablar de representaciones sociales y de multiplicidad de las mismas da cuenta de memorias encuadradas pero múltiples, en sus desarrollos no se encuentra una idea de algo así como una memoria colectiva única y compartida socialmente.

Respecto al segundo interrogante planteado en este apartado, para avanzar en una posible respuesta que se articule a la permanencia en el tiempo de determinados acontecimientos, resulta sugestiva la noción de "realización simbólica del genocidio" planteada por el sociólogo Daniel Feierstein. El autor la ubica como la fase posterior al aniquilamiento (el está pensando el caso argentino como un genocidio pero podemos trasladar la definición al "pos" de cualquier experiencia límite a decir de Pollak), en donde se clausuran las relaciones sociales encarnadas por aquellos que fueron asesinados, reorganizando las relaciones sociales a partir de los modos de narrar y representar la experiencia represiva. La realización simbólica no implica el olvido del terror sino que a partir de los modos en que se representa esta experiencia se generan diversos modos de comprender este pasado vinculado al terror desde el presente (Feierstein, 2012).

Incorporando las aproximaciones delineadas hasta aquí se puede plantear que estos modos de comprender el pasado vinculado al terror desde el presente se articula a los intereses de la multiplicidad de actores que definen distintas representaciones y sentidos de lo acontecido. 


\section{Cuarta aproximación. Pasado, presente, futuro}

Un último aporte que es fundamental para definir un concepto de memoria vinculado a los propios intereses de investigación, es el que realiza Elizabeth Jelin. Retomando a Maurice Halbwachs (2005) -a partir de una analogía respecto de los cuadros sociales de la memoria- sostendrá que ésta es un mecanismo cultural tendiente a fortalecer el sentido de pertenencia de la comunidad o grupo. El elemento distintivo que incorpora esta autora y que se encuentra estrechamente vinculado al contexto latinoamericano en el que está pensando su concepto de memoria como trabajo (como una operación de dar sentido al pasado), es la memoria vinculada no solo al recuerdo sino en un estrecho vinculo con el olvido. Este planteo es estratégico ya que Jelin construye su concepto en función de articularlo a acontecimientos traumáticos, de carácter represivo y que incluyen al aniquilamiento como forma de operar-justamente en el contexto de dictaduras latinoamericanas-. Lo más valioso de su aporte es el modo en que vincula temporalidad e intención de las memorias; en esta operación de dar sentido al pasado desde el presente esta articulación se establece en función de un futuro deseado. Entonces, la memoria tendría su temporalidad específica en el presente pero esta sería un nexo entre pasado y futuro.

La temporalidad específica es el presente ya que éste irá cambiando, y estos cambios son lo que proveerán de nuevos marcos interpretativos para comprender el pasado. En esta misma línea -complementariamente a los marcos interpretativos de Jelin-, en posible ubicar la noción de "régimen de memoria" de Emilio Crenzel a la que se hizo referencia al comienzo de este trabajo y que también es sumamente sugerente para delimitar un concepto propio de memoria. Esta comprensión del pasado desde el presente es un "trabajo" ya que coloca a la sociedad y sus distintos actores en un lugar activo y transformador otorgándole una especificidad política de "emprender" la memoria vinculada a trabajar en pos del futuro (Jelin, 2002).

\section{Discusión}

\section{Delineando una definición de memoria articulable a la prensa como objeto de indagación}

Esta articulación se realiza con base en resultados de investigación provisorios del análisis del trabajo de archivo. Decimos "provisorios" ya que para construir el concepto de memoria recurrimos al trabajo de archivo realizado en relación a la cobertura del juicio a Etchecolatz que consta de una totalidad de 103 notas periodísticas, crónicas y editoriales relacionadas a los juicios; 23 corresponden a Clarín y 80 a Página 12. La cantidad de notas de uno y otro diario también es importante a la hora de dar cuenta de la importancia atribuida a determinados procesos sociales por uno y otro medio.

A partir del recorrido realizado en los apartados anteriores y de la incorporación de las perspectivas de cada uno de los cuatro abordajes, se puede trazar una definición propia de memoria a los fines de que sea útil en tanto herramienta, no sólo teórica sino también metodológica (Rousso, 1991) para la investigación propia. En este sentido, a continuación se presentará a modo de discusión una serie de elementos presentes en las notas periodísticas de los diarios Página 12 y Clarín sobre el juicio al represor Miguel Etchecolatz. Este proceso judicial es el primero en desarrollarse luego de la declaración de inconstitucionalidad de las leyes de impunidad. En el mismo se juzgó al represor por delitos cometidos contra 14 víctimas. Etchecolatz ofició el cargo de Director de Investigaciones de la Policía de Buenos Aires entre marzo de 1976 y fines de 1977 siendo la mano derecha del jefe de la policía de Buenos Aires Ramón Camps durante este período. Fue condenado a prisión perpetua por el homicidio calificado de Diana Teruggi y la privación ilegítima de la libertad, tormentos y homicidio calificado de Patricia Dell'Orto, Nora Formiga, Elena Arce, Ambrosio de Marco y Margarita 
Delgado en un conjunto de centros clandestinos de detención del sur de la provincia de Buenos Aires conocidos como "Circuito Camps". En el marco de las audiencias del juicio, Jorge Julio López fue un testigo que en calidad de sobreviviente aportó gran cantidad de información que involucraba a Etchecolatz en la comisión de los graves crímenes. El 18 de septiembre de 2006, a poco de finalizar el juicio, se leerían los alegatos. Ese día López fue desaparecido por segunda vez, hasta la fecha no hay datos certeros sobre su paradero.

En lo que refiere al "encuadramiento" es posible señalar que el mismo se da a partir de la nueva coyuntura posibilitada por la anulación de la legislación de impunidad, la apertura de los juicios y el impulso político de los mismos por parte del estado. También hay que destacar que cada diario tiene un encuadramiento propio, el cual excede ampliamente al carácter ejemplificativo de este apartado pero podemos señalarlo dentro del marco de la "multiplicidad" que caracteriza a la memoria.

Dentro de lo que se señaló como "multiplicidad", "fragmentariedad" e "incoherencia" que caracteriza a los modos en que se articulan las memorias, se indica como distintivo de ambos periódicos que desde el inicio de las crónicas en junio de 2006 se encuentran relatos heterogéneos y diferenciales respecto al proceso judicial y a los actores que intervienen en el mismo. A modo de referencia, esto puede observarse, por ejemplo, a partir de un indicador analítico, la enunciación indiferenciada: para referir al juicio se hace uso de múltiples conceptos que intentan dar cuenta del mismo, pertenecen al mismo registro (ya que se relacionan al proceso judicial) pero no configuran exactamente lo mismo. Otro elemento lo constituye la falta de una definición precisa, no existe coherencia interna en las formas de enunciar a lo largo de todo el corpus que comprende los artículos de cobertura del juicio. En ambos diarios el proceso judicial es tematizado de forma divergente aludiendo a distintas nociones; "derechos humanos", "fuerzas armadas", "delitos contra la humanidad", "anulación de leyes del perdón”, "juicio oral contra un represor", "crímenes de la última dictadura", entre los más destacados. El día que inicia el juicio, el 20 de junio de 2006, ambos periódicos publican artículos sobre el juicio pero no titulan sobre lo que se está juzgando sino que se hace referencia a la coyuntura más amplia en la que se encuadra el proceso judicial, el juicio es una consecuencia directa de la anulación de las leyes de impunidad. Clarín titula "Primer juicio a un represor tras la anulación de las leyes de impunidad" y Página 12: "Primer juicio después de la anulación de las leyes de Obediencia Debida y Punto Final" .

Es recién luego de la lectura de la sentencia que ambos medios denominan a los hechos bajo una misma definición que coincide con la adoptada en la sentencia, la de genocidio. El 20 de septiembre de 2006, titulan; Clarín: "Condenan a reclusión perpetua a Etchecolatz por genocida", y Página 12: "Delitos cometidos en el marco del genocidio". Es decir, la sentencia tiene efectos performativos sobre la construcción de representaciones de los medios que posibilita la conformación de nuevos marcos o cuadros de lo representable. En este sentido, retomando a Jelin (2002) es posible identificar al campo jurídico como un emprendedor de memoria:

El emprendedor es un generador de proyectos, de nuevas ideas y expresiones, de creatividad -más que de repeticiones-. La noción remite a la existencia de una organización ligada al proyecto de memoria, que puede implicar jerarquías sociales, mecanismos de control y de división del trabajo bajo el mando de estos emprendedores. (p. 48)

Otros elementos que surgen -impensables de surgir en las anteriores coyunturas-, y que ingresan en este nuevo régimen de memoria como representables por parte de los diarios son los delitos sexuales y la complicidad civil $^{7}$. Vale destacar que teniendo en cuenta la característica de multiplicidad y fragmentariedad de la memoria, estos hechos solo aparecen en uno de los diarios (Diario Página 12).

\section{Conclusiones}

Finalizando el trabajo, se destaca que el objetivo del mismo fue presentar un concepto de memoria

\footnotetext{
7. Ver Página 12. Sobre complicidad civil, 18 de julio de 2006: "La complicidad del silencio". Sobre delitos sexuales en el marco del cautiverio, 31 de agosto de 2006: "Violaciones sistemáticas".
} 
que no implique la intención de crear materialidad (como lo plantea Nora) y permanencia sino que principalmente se buscó destacar al mismo a partir del conflicto presente por un retorno de cuestiones no saldadas respecto del pasado. Es por ello que se retomaron principalmente aquellos aportes que dan cuenta de los encuadramientos o contextos en los que emergen determinadas representaciones y memorias -que son múltiples-, que las mismas se vinculan a experiencias límite que perviven a lo largo de los años y que son móviles en función de los valores que poseen los distintos grupos sociales en el presente.

Un interrogante factible de plantear, que excede este trabajo pero puede delinearse para futuras indagaciones es; ¿puede ser considerado el discurso periodístico como un vector de memoria que intenta reconstruir a partir del presente lo traumático que retorna en los procesos judiciales? ¿Puede considerarse a los juicios como un cierre o tramitación, una elaboración de este pasado que permanece sin ser saldado?

En principio sostenemos que es factible, ya que los medios de comunicación son un escenario privilegiado para el despliegue y difusión de representaciones y sentidos a partir de lenguajes y formas de narrar los procesos sociales traumáticos y los actores intervinientes en los mismos (víctimas, perpetradores, testigos, magistrados). Además proveen elementos para la configuración de nuevos marcos de lo memorable, en este sentido la incorporación de la calificación adoptada en la sentencia judicial es un claro ejemplo. Por último la memoria es una práctica social que requiere de soportes materiales y simbólicos y se debe avanzar en la indagación de este último componente.

\section{Referencias bibliográficas}

Feld, C. (2012). La televisión ante el pasado reciente: ¿Cómo estudiar la relación entre TV y memoria social? Anos 90, 19(36), 149-172.

Feierstein, D. (2007). El genocidio como práctica social. Entre el nazismo y la experiencia argentina. Buenos Aires: Fondo de Cultura Económica.
Feierstein, D. (2012). Memorias y Representaciones. Sobre la elaboración del genocidio. Buenos Aires: Fondo de Cultura Económica.

Gatti, G. (2008). El detenido-desaparecido. Narrativas posibles para una catástrofe de la identidad. Montevideo: Trilce.

Halbwachs, M. (2005). Memoria individual y memoria colectiva. Estudios, 16, 163-187.

Jelin, E. (2002). Los trabajos de la memoria. Madrid y Buenos Aires: Siglo XXI.

Lacapra, D. (2007). Historia en tránsito. Experiencia, identidad, teoría crítica. Buenos Aires: Fondo de Cultura Económica.

Lavabre, M. (1998). Maurice Halbwachs et la sociologie de la mémoire. Raison Présente, 128, 47-56.

Namer, G. (1998). Antifascismo y la memoria de los músicos de Halbwachs. Ayer, 32, 35-56.

Nora, P. (2008). Entre memoria e historia. La problemática de los lugares. En N. Pierre. (Ed.), Pierre Nora en Les lieux de mémoire (pp.9-33). Montevideo: Trilce.

Nora, P. (1998). "La aventura de Lieux de mémoire". Ayer, 32, 17-34.

Pollak, M. (2006). Memoria, olvido, silencio. La Plata: Al Margen Editora.

Portelli, A. (2003). Memoria e identidad. Una reflexión desde la Italia postfascista. En E. Jelin \& V. Langland (Comps), Monumentos, memoriales y marcas territoriales (pp. 165-190). Madrid y Buenos Aires: Siglo XXI.

Rousso, H. (2000). "El duelo es imposible y necesario". Revista Puentes, 2, 30-40.

Rousso, H. (2012). "Para una historia de la memoria colectiva: el post Vichy". Aletheia, 3(5), 1-14. Recuperado de http://www.aletheia.fahce.unlp.edu. ar/numeros/numero-5/pdfs/Rousso-ok.pdf

Traverso, E. (2012). La historia como campo de batalla: interpretar las violencias del siglo XX. Buenos Aires: Fondo de Cultura Económica. 